Delft University of Technology

\title{
Thermo-elastic optical coherence microscopy
}

Doug Deen, Aaron; Pfeiffer, Tom; Van Beusekom, Heleen; Essers, Jeroen; Huber, Robert; Van Der Steen, Antonius F.W.; Van Soest, Gijs; Wang, Tianshi

DOI

10.1117/12.2550998

Publication date

2020

Document Version

Final published version

Published in

Proceedings of SPIE

\section{Citation (APA)}

Doug Deen, A., Pfeiffer, T., Van Beusekom, H., Essers, J., Huber, R., Van Der Steen, A. F. W., Van Soest, G., \& Wang, T. (2020). Thermo-elastic optical coherence microscopy. In J-X. Cheng, W. Min, \& G. J.

Simpson (Eds.), Proceedings of SPIE: Progress in biomedical optics and imaging (Vol. 11252). [112520H] SPIE. https://doi.org/10.1117/12.2550998

\section{Important note}

To cite this publication, please use the final published version (if applicable).

Please check the document version above.

\section{Copyright}

Other than for strictly personal use, it is not permitted to download, forward or distribute the text or part of it, without the consent of the author(s) and/or copyright holder(s), unless the work is under an open content license such as Creative Commons.

\section{Takedown policy}

Please contact us and provide details if you believe this document breaches copyrights.

We will remove access to the work immediately and investigate your claim. 


\section{Thermo-elastic optical coherence microscopy}

Doug Deen, Aaron, Pfeiffer, Tom , van Beusekom, Heleen, Essers, Jeroen, Huber, Robert, et al.

Aaron Doug Deen, Tom Pfeiffer, Heleen van Beusekom, Jeroen Essers, Robert Huber, Antonius F. W. van der Steen, Gijs van Soest, Tianshi Wang, "Thermo-elastic optical coherence microscopy," Proc. SPIE 11252, Advanced Chemical Microscopy for Life Science and Translational Medicine, $112520 \mathrm{H}$ (21 February 2020); doi: 10.1117/12.2550998

SPIE. Event: SPIE BiOS, 2020, San Francisco, California, United States 


\title{
Thermo-elastic optical coherence microscopy
}

\author{
Aaron Doug Deen ${ }^{1}$, Tom Pfeiffer ${ }^{2}$, Heleen van Beusekom ${ }^{1}$, Jeroen Essers ${ }^{1}$, Robert Huber ${ }^{2}$, \\ Antonius F.W. van der Steen ${ }^{1,3,4}$, Gijs van Soest ${ }^{1}$, and Tianshi Wang,,* \\ ${ }^{1}$ Erasmus University Medical Center, P.O. Box 2040, Rotterdam 3000 CA, The Netherlands \\ ${ }^{2}$ Institut für Biomedizinische Optik, Universität zu Lübeck, Peter-Monnik-Weg 4, 23562 Lübeck Germany \\ ${ }^{3}$ Shenzhen Institutes of Advanced Technology, Chinese Academy of Sciences, 518055 Shenzhen, China \\ ${ }^{4}$ Department of Imaging Science and Technology, Delft University of Technology, Delft 2600 AA, The Netherlands \\ ${ }^{*}$ Corresponding author: T. Wang, Email: t.wang.1@erasmusmc.nl
}

\begin{abstract}
The absorption of laser pulses by tissue leads not only to the generation of acoustic waves, but also to nanometer to sub-micrometer scale displacement. After the initial expansion, a quasi-steady state is achieved in a few microseconds. Previously we introduced the concept of thermo-elastic optical coherence tomography (TE-OCT) to "visualise" the rapid thermo-elastic expansion by measuring the Doppler phase shift rather than "listening" to the acoustic wave as in photoacoustic imaging. In this study, we built a microscopic setup for high-speed 3D TE-OCT imaging, by means of thermo-elastic optical coherence microscopy (TE-OCM). The repetition rate of pulsed laser was set to $100 \mathrm{~Hz}$ and the line rate of the OCT system is $1.5 \mathrm{MHz}$. The OCT beam and the laser pulse were focused upon the same location on the sample FWHM spot sizes of $300 \mu \mathrm{m}$ for the pulsed laser and $40 \mu \mathrm{m}$ FWHM for the OCT beam. For each laser pulse, an M-mode OCT image consisting of 90 A-lines was acquired. The Doppler phase shift was extracted by comparing the phase signal before and after the pulse arrival. Within 6 minutes, a 3D TE-OCM image $\left(10 \times 10 \times 4 \mathrm{~mm}^{3}\right)$ can be acquired and processed. Imaging experiments were carried out in swine meat using $1210 \mathrm{~nm}$ excitation wavelength to highlight lipid in tissue. The results show that no significant displacement was detected in swine muscle while strong displacement was observed in lipid, owing to the optical absorption features. Furthermore, fatty tissue is easily identified in the 3D TE-OCM image while the conventional OCT images provides the structural information.
\end{abstract}

\section{INTRODUCTION}

Optical coherence tomography (OCT) is an imaging modality which provides structural information of tissue samples with micrometer scale resolution. ${ }^{1}$ Phase-sensitive OCT is an extension of OCT which measures tissue displacement at the nanometer to sub-micrometer scale by processing the Doppler phase shift. Depending on the nature of excitation, functional images can be reconstructed based on the phase-sensitive OCT technique. For instance, optical coherence elastography maps tissue stiffness by utilising mechanical stimulus while photothermal OCT visualises optical absorption by employing continuous wave lasers. ${ }^{2}, 3$ Previously, we introduced the concept of "Thermo-elastic optical coherence tomography (TE-OCT)". ${ }^{4}$ Rather than "listening" to the acoustic wave by use of an ultrasound transducer as in photoacoustic imaging, TE-OCT "visualises" the thermo-elastic displacement by measuring the Doppler OCT phase shift. Using a forward-looking probe to scan the sample, cross-sectional TE-OCT images were acquired which provides tissue type information in addition to the tissue morphology offered by conventional OCT.

The absorption of short laser pulses, leads not only to the generation of acoustic waves but also to minute tissue expansions on the scale of tens of nanometers. Such tissue expansion, known as thermo-elastic deformation occurs within a few microseconds after pulse incidence, then achieves a quasi-steady state. ${ }^{5}$ In a linear and isotropic material, one-dimensional thermoelastic displacement $u_{(z, t)}$ can be determined by

$$
\frac{\delta^{2} u}{\delta z^{2}}-\frac{1}{c_{L}^{2}} \frac{\delta^{2} u}{\delta t^{2}}=-\sqrt{\frac{\mu_{a}}{3 \mu_{a t t}}} \frac{P \beta}{3 \rho C_{p}} \frac{(1+v)}{(1-v)} \exp \left(-\sqrt{3 \mu_{a} \mu_{a t t}} \cdot z\right)
$$

\footnotetext{
Advanced Chemical Microscopy for Life Science and Translational Medicine, edited by

Ji-Xin Cheng, Wei Min, Garth J. Simpson, Proc. of SPIE Vol. 11252, 112520H

(c) 2020 SPIE · CCC code: 1605-7422/20/\$21 - doi: 10.1117/12.2550998
}

Proc. of SPIE Vol. $11252112520 \mathrm{H}-1$ 
where $\beta$ is the thermal coefficient of volume expansion, $\rho$ is the mass density, $c_{L}$ is the longitudinal speed of sound, $\mu_{a}$ and $\mu_{a t t}$ are the optical absorption and optical attenuation coefficients respectively, $v$ is Poisson's ratio, $C_{p}$ is the heat capacity and $P$ is the optical fluence.

From this an analytical solution for displacement in the quasi-steady state then follows

$$
\begin{aligned}
u(z, t) & =u_{o}\left\{\exp \left[-\sqrt{3 \mu_{a} \mu_{a t t}} \cdot c_{L}\left(t-\frac{z}{c_{L}}\right)\right]+\exp \left[-\sqrt{3 \mu_{a} \mu_{a t t}} \cdot c_{L}\left(t+\frac{z}{c_{L}}\right)\right]-2 \exp \left(-\sqrt{3 \mu_{a} \mu_{a t t}} \cdot z\right)\right\} \\
& \approx-2 u_{o} \exp \left(-\sqrt{3 \mu_{a} \mu_{a t t}} \cdot z\right),
\end{aligned}
$$

where $u_{o}$ is given as:

$$
u_{o}=\sqrt{\frac{\mu_{a}}{3 \mu_{a t t}}} \frac{(1+v)}{(1-v)} \frac{\beta}{C_{p}} \frac{P}{3 \rho},
$$

It can be seen that the surface displacement is related to many properties of the tissue, however, it is mainly dominated by optical absorption. ${ }^{6}$ The surface thermo-elastic displacement in an homogeneous sample can be obtained by comparing the phase-shift prior to and following laser irradiation by

$$
u=\Phi \frac{\lambda_{o}}{4 \pi n}
$$

where $\Phi$ is the Doppler OCT phase shift, $n$ is the refractive index of the sample and $\lambda_{o}$ is the centre wavelength of the OCT laser source. ${ }^{4}$ In this study, we advance the TE-OCT technology and build a microscopic setup, by means of thermo-elastic optical coherence microscopy (TE-OCM). Using TE-OCM, we aim to achieve high speed, label-free and free space chemical microscopic imaging.

\section{METHOD}

\subsection{Imaging system}

The schematic diagram of the TE-OCM setup is shown in Fig.1. The tunable pulsed laser (Innolas, SpitLight EVO-OPO, Germany), has a wide wavelength tuning range of $660-2600 \mathrm{~nm}$ with pulse durations of $4-7 \mathrm{~ns}$ and repetition rates of up to $200 \mathrm{~Hz}$. The phase sensitive OCT system is based on a $1.5 \mathrm{MHz}$ Fourier domain mode locked (FDML) laser with a central wavelength of $1310 \mathrm{~nm}$ and a tuning range of $90 \mathrm{~nm} .{ }^{7}$ The performance parameters of the OCT system are; an output power of $20 \mathrm{~mW}$, a sensitivity of $102 \mathrm{~dB}$, an imaging depth of 4.0 $\mathrm{mm}$ in air and a phase stability of $0.5 \mathrm{~nm}$ in air. In the OCT system, $90 \%$ of the output power from the FDML is sent to the sample arm, while the remaining $10 \%$ is sent to the reference arm. The reflected light from the sample is recombined with the light from the reference arm in a 50/50 coupler and sent to a $1.6 \mathrm{GHz}$, InGaAs balanced photodetector (Thorlabs, PDB480C-AC, U.S.). The photodetector is combined with a 4 GS/s, 8-bit data acquisition board and used to record the interferogram. The pulsed laser and the OCT beams were coupled and focused upon the same location on the sample with full width half maximum (FWHM) spot sizes of 300 $\mu \mathrm{m}$ (pulsed laser) and $40 \mu \mathrm{m}$ (OCT). An x,y,z translation stage setup (Aerotech, ACT115DL, U.S; Thorlabs, KMTS50E/M, U.S; Newport, LTA-HS, U.S) was used to move the sample to realise beam scanning.

\subsection{Signal processing}

For each laser pulse, an M-mode OCT image consisting of 90 A-lines $(\approx 60 \mu \mathrm{s})$ was acquired. The acquisition of each M-mode image was triggered $10 \mu \mathrm{s}$ prior to the arrival of the pulse. The phase signal of the OCT dataset was compared prior to and after the arrival of the pulse in order to extract the Doppler phase shift and thus measure the displacement. For each M-mode image, the Doppler phase shift A-lines were created and used to construct the 3D TE-OCM image, while the conventional OCT images can be provided simultaneously. 

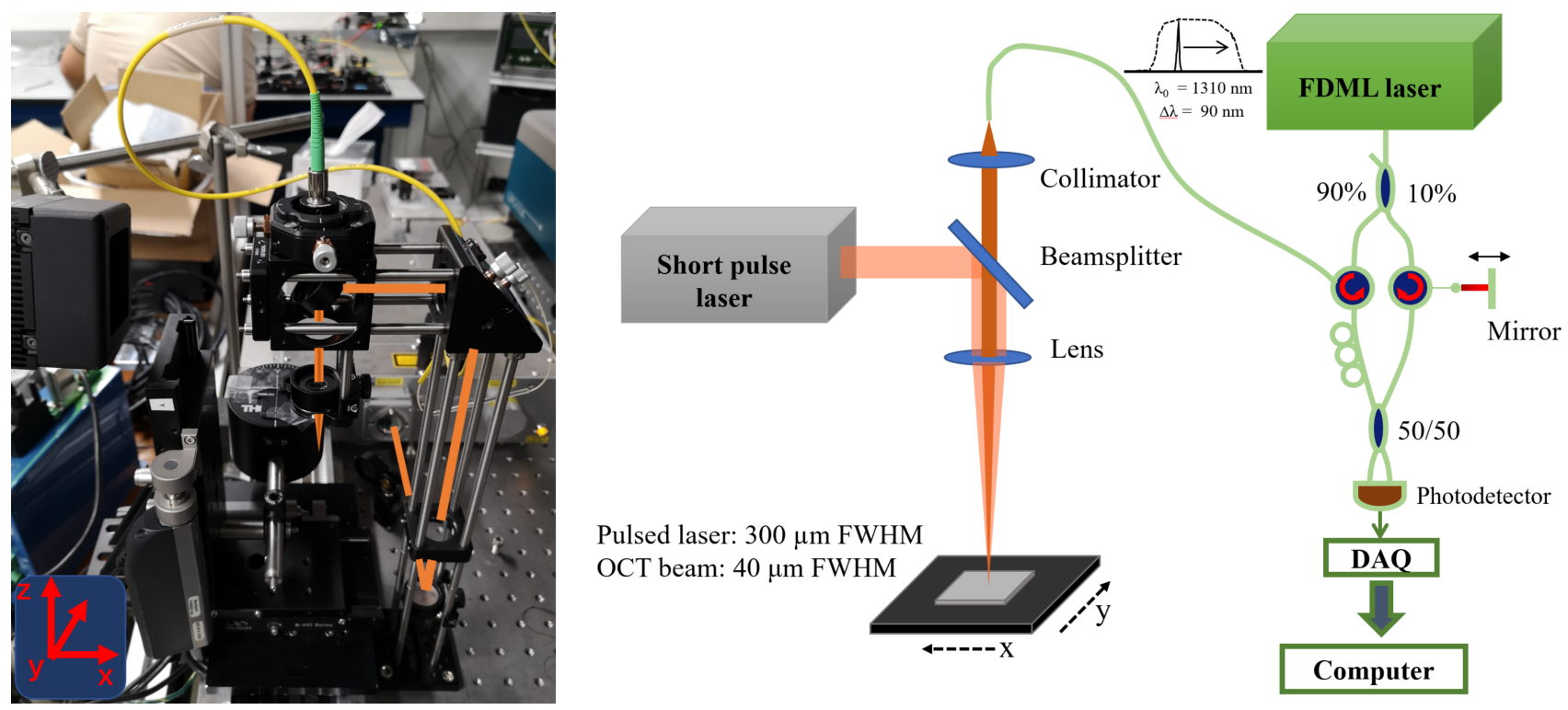

Figure 1. Photo and schematic diagram of TE-OCM system. The pulsed laser and OCT beams are aligned and focused on the sample at the same point. A 3D translational stage setup was used to realise beam scanning. FDML: Fourier domain mode locked, DAQ: Data acquisition card.

\section{RESULTS}

\subsection{Beam profile and M-mode images}

The two beams were first aligned using a beam profiler (BP209-IR2/M, Thorlabs, U.S.) with the OCT beam centred in the pulsed laser beam as seen in Fig.2(a). Parts (b) and (c) of Fig.2 shows M-mode images consisting of 90 A-lines of muscle and lipid respectively. The pulse laser was operated at $1210 \mathrm{~nm}$ wavelength. The yellow arrow indicates the arrival of a single pulse from the pulsed laser. No changes can be observed in the conventional OCT images following pulse incidence as shown in Fig.2 (b) and (c), whereas a significant displacement is observed in the phase-shift image of lipid Fig.2(e), owing to the strong optical absorption of lipid at $1210 \mathrm{~nm}$ wavelength and sufficient excitation energy. However, no significant displacement was detected in the muscle as a result of its low optical absorption.
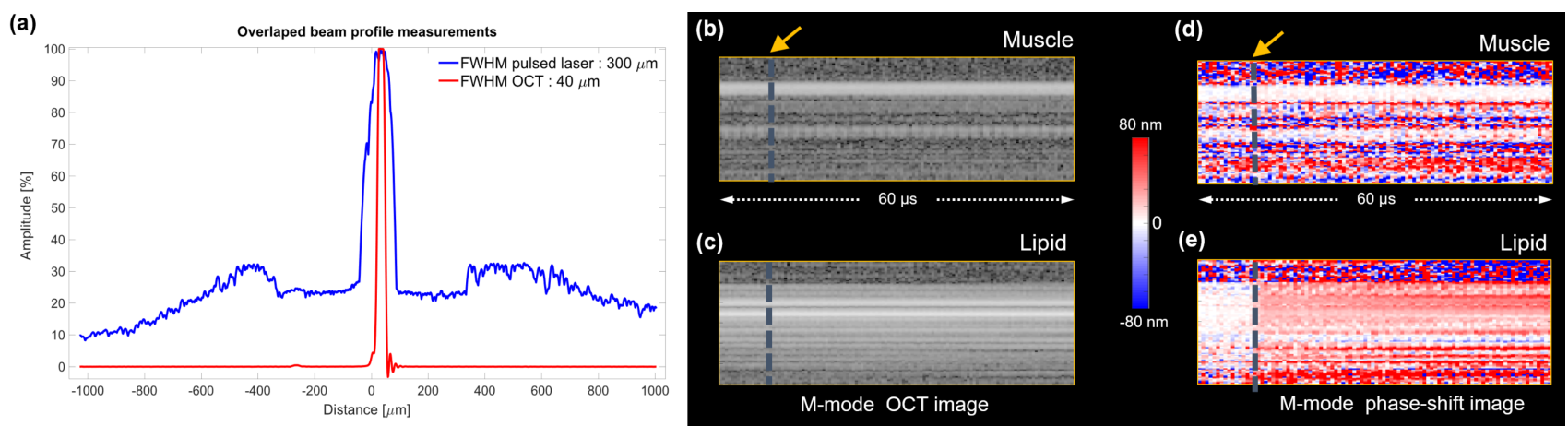

Figure 2. Beam profiles and M-mode OCT images. Image (a) shows the measured beam profiles of both the pulsed laser and OCT beams. (b) and (c) shows m-mode images of muscle and lipid respectively. (d) and (e) show m-mode Doppler phase-shifted images, with a positive surface displacement observed in (e) following pulse arrival. Yellow arrows indicate laser pulse arrival. 


\subsection{Thermo-elastic displacement spectrum}

We characterised the thermo-elastic spectrum of lipid and muscle, by tuning the excitation wavelength of the pulsed laser from 1150 to $1250 \mathrm{~nm}$, in steps of $1 \mathrm{~nm}$. This wavelength range was chosen specifically to identify lipid since it is known that lipid has a strong optical absorption peak at $1210 \mathrm{~nm}$. The repetition rate was set to $10 \mathrm{~Hz}$ with a pulse duration of $5 \mathrm{~ns}$ and a power of $1.8 \mathrm{~mJ}$ per pulse. For each step, a single laser pulse was sent and the surface displacement measured in a M-mode image. For each spectrum we acquired and averaged over three individual spectrums for both lipid and muscle. As shown in Fig.3, the thermo-elastic spectrum of lipid shows similar features to that of the optical absorption spectrum, which is mainly due to the thermo-elastic deformation being dominated by optical absorption. Conversely, the magnitude of thermo-elastic deformation of muscle is much smaller than lipid at this wavelength range, owning to its relatively low optical absorption.

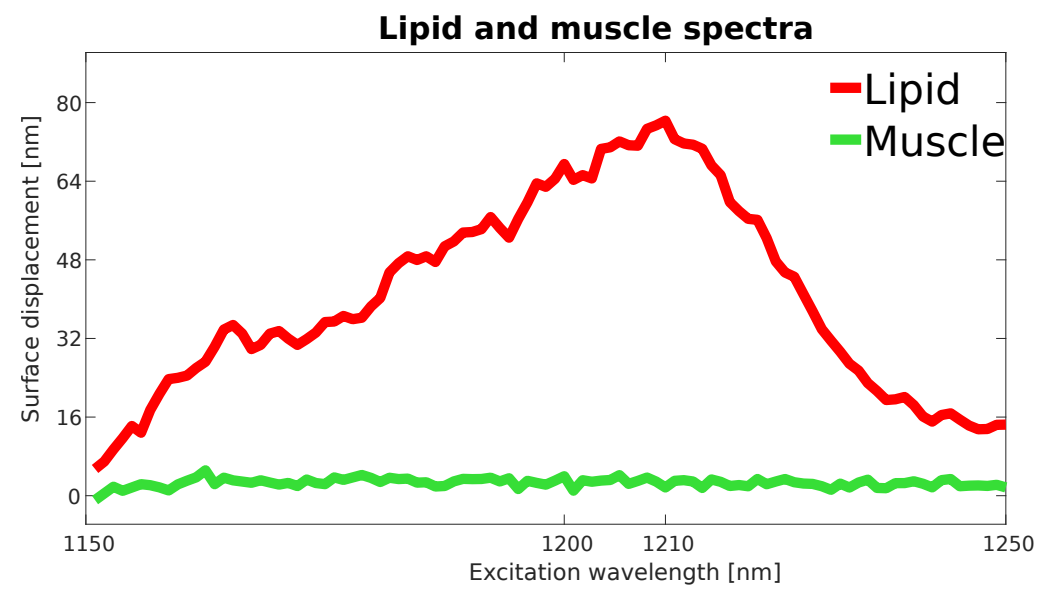

Figure 3. Thermo-elastic displacement spectrums. Acquired spectrums of lipid and muscle illustrating a wavelength scan of $1150-1250 \mathrm{~nm}$ with corresponding measured surface displacement.

\subsection{Microscopic TE-OCT imaging}

3D TE-OCM images were acquired in swine meat using the microscopic setup. Experimentally, a 3D image of a slice of swine meat with dimensions $7.5 \times 10 \times 4 \mathrm{~mm}$ was imaged. The pulsed laser was operated at $100 \mathrm{~Hz}$ with a pulse duration of $5 \mathrm{~ns}$ and a power $0.24 \mathrm{~mJ}$ per pulse on the sample, in order to ensure a detectable thermoelastic expansion signal while preventing tissue ablation. Fig.4(a) shows the en face view of the OCT image which offers the conventional structural information of the meat, however the tissue type, i.e. lipid or muscle cannot be distinguished. Fig.4(b) shows the TE-OCM image, in which the red areas indicates the presence of lipid and the white areas indicates the presence of muscle. A surface displacement is clearly observed in the area containing lipid due to its strong optical absorption at $1210 \mathrm{~nm}$ while the highlighted area containing muscle, shows no change in displacement. Thus, tissue type information is now provided in addition to the structural information offered by conventional OCT. 


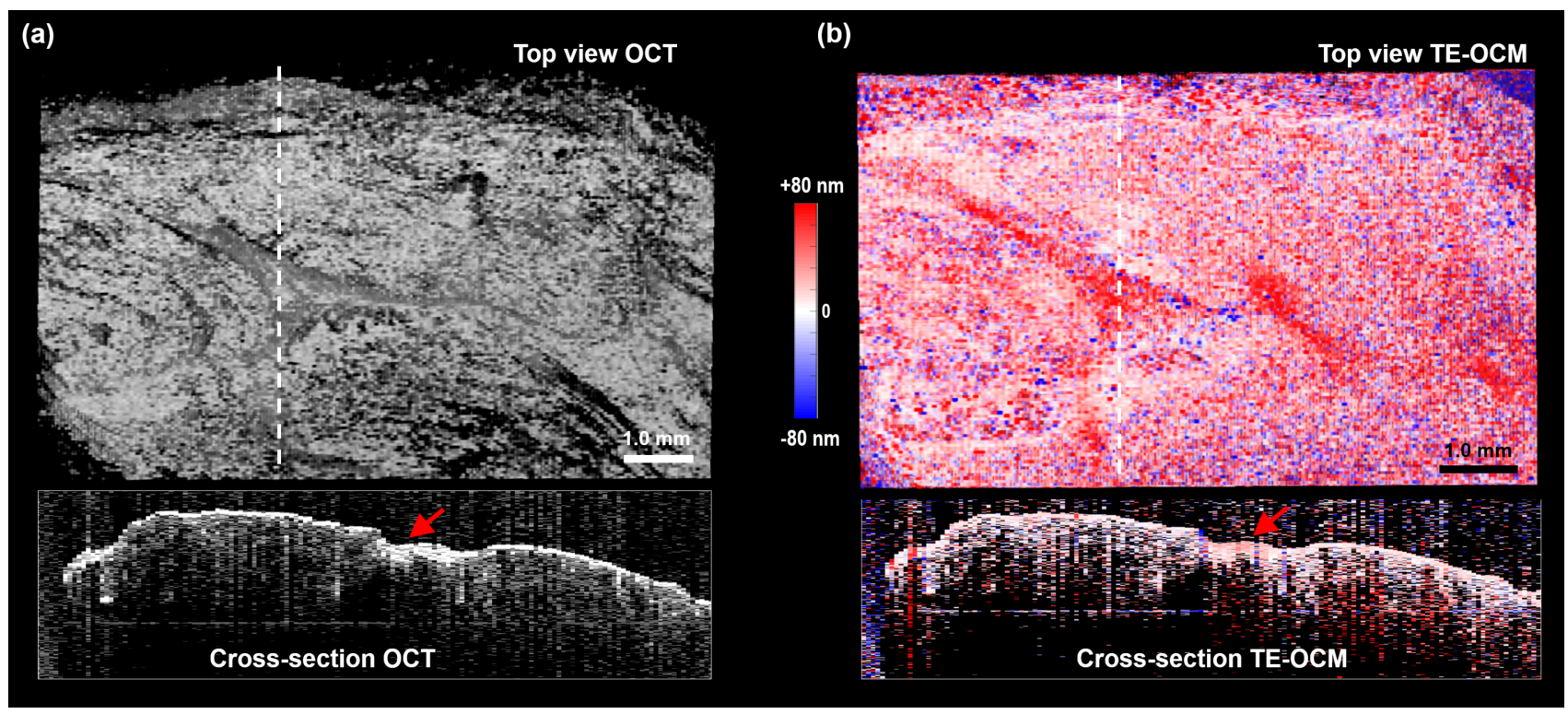

Figure 4. OCT image of swine meat. En face views of the OCT images showing morphological and phase information are seen in (a) and (b) respectively. The red arrows on the cross-sectional images indicate the location of lipid tissue.

\section{DISCUSSION}

Owing to the unique thermo-elastic properties of the swine lipid and muscle, imaging contrast is offered by the TE-OCM image, which allows easy identification of the lipid tissue. Together with the structural OCT images, TE-OCM visualises both the tissue type and morphology simultaneously with micro-scale level resolution. It is worth mentioning that TE-OCM requires no additional exogenous contrast agents and performs fast imaging in free space. Sample preparation is also simple and quick. Therefore, we believe that TE-OCM holds promise as an advanced chemical microscope. By choosing the proper excitation wavelength, TE-OCM can potentially be used to detect many other types of tissue other than lipid.

As a limitation of this study, the output power of the pulsed laser varies widely (on average $+/-20 \%$ ), which affects the surface displacement measurement and induces noise in the images. In the future, we will develop real time monitoring of the pulsed laser and implement power compensation for the surface displacement. At this moment, we consider the resolution of TE-OCM (from the En face view) to be the FWHM of the OCT beam, which is the same as the case of the acoustic resolution of photoacoustic imaging, i.e. the detection beam spot is much smaller than the excitation beam spot. In future work, we will carry out experimental characterisation of the imaging resolution. In this study, the image acquisition time is determined by the repetition rate of the pulsed laser. Limited by the $100 \mathrm{~Hz}$ rate, TE-OCM acquires an area of $(10 \mathrm{~mm} \times 10 \mathrm{~mm})$ within 6 minutes. In the future we will increase the repetition rate to $200 \mathrm{~Hz}$ thereby decreasing the image acquisition time to $\approx 3$ minutes.

\section{CONCLUSION}

In this study, we demonstrate the first microscopic realisation of the TE-OCT technique, by means of TEOCM. TE-OCM achieves high speed, label-free and free space microscopic imaging, simultaneously providing the chemical and morphological information of the sample. We believe that TE-OCM can potentially bring new opportunities to diagnostic pathological imaging and be utilised as an imaging tool for biomedical research. 


\section{REFERENCES}

[1] Drexler, W., M.Liu, Kumar, A., Kamali, T., Unterhuber, A., and Leitgeb, R., "Optical coherence tomography today: speed, contrast, and multimodality," Journal of Biomedical Optics 19(7), 071412-1 - 071412-34 (2014).

[2] Kennedy, B. F., Kennedy, K. M., and Sampson, D., "A review of optical coherence elastography: Fundamentals, techniques and prospects," IEEE Journal of Selected Topics in Quantum Electronics 20(2), 7101217, $1-17$ (2014).

[3] Skala, M. C., Crow, M. J., Wax, A., and Izatt, J. A., "Photothermal optical coherence tomography of epidermal growth factor receptor in live cells using immunotargeted gold nanospheres," Nano Letters 8(10), 3461-3467 (2008).

[4] Wang, T., Pfeiffer, T., Wu, M., Wieser, W., Amenta, G., Draxinger, W., van der Steen, A. F. W., Huber, R., and van Soest, G., "Thermo-elastic optical coherence tomography," Optics Letter 42, 3466 - 3469 (2017).

[5] Soroushian, B., Whelan, W. M., and Kolios, M. C., "Study of laser-induced thermoelastic deformation of native and coagulated ex-vivo bovine liver tissues for estimating their optical and thermomechanical properties," Journal of Biomedical Optics 15(6), 065002-1 - 065002-10 (2010).

[6] J. Xia, J. Y. and Wang, L. V., "Photoacoustic tomography: principles and advances," Progress in Electromagnetics Research 147, 1-22 (2014).

[7] Huber, R., Wojtkowski, M., and Fujimoto, J. G., "Fourier domain mode locking (fdml): A new laser operating regime and applications for optical coherence tomography," Optics Expresss 14(8), 3225-3237 (2006). 